\title{
BAR-encapsulated nanoparticles for the inhibition and disruption of Porphyromonas gingivalis-Streptococcus gordonii biofilms
}

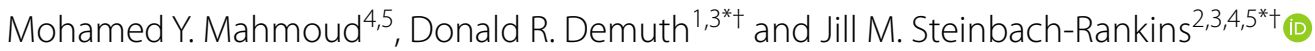

\begin{abstract}
Background: Porphyromonas gingivalis adherence to oral streptococci is a key point in the pathogenesis of periodontal diseases (Honda in Cell Host Microbe 10:423-425, 2011). Previous work in our groups has shown that a region of the streptococcal antigen denoted BAR (SspB Adherence Region) inhibits P. gingivalis/S. gordonii interaction and biofilm formation both in vitro and in a mouse model of periodontitis (Daep et al. in Infect Immun 74:5756-5762, 2006; Daep et al. in Infect immun 76:3273-3280, 2008; Daep et al. in Infect Immun 79:67-74, 2011). However, high localized concentration and prolonged exposure are needed for BAR to be an effective therapeutic in the oral cavity.
\end{abstract}

Methods: To address these challenges, we fabricated poly(lactic-co-glycolic acid) (PLGA) and methoxy-polyethylene glycol PLGA (mPEG-PLGA) nanoparticles (NPs) that encapsulate BAR peptide, and assessed the potency of BARencapsulated NPs to inhibit and disrupt in vitro two-species biofilms. In addition, the kinetics of BAR-encapsulated NPs were compared after different durations of exposure in a two-species biofilm model, against previously evaluated BAR-modified NPs and free BAR.

Results: BAR-encapsulated PLGA and mPEG-PLGA NPs potently inhibited biofilm formation (IC50 = $0.7 \mu \mathrm{M})$ and also disrupted established biofilms (IC50 $=1.3 \mu \mathrm{M})$ in a dose-dependent manner. In addition, BAR released during the first $2 \mathrm{~h}$ of administration potently inhibits biofilm formation, while a longer duration of $3 \mathrm{~h}$ is required to disrupt preexisting biofilms.

Conclusions: These results suggest that BAR-encapsulated NPs provide a potent platform to inhibit (prevent) and disrupt (treat) P. gingivalis/S. gordonii biofilms, relative to free BAR.

Keywords: Polymer nanoparticle, Poly(lactic-co-glycolic acid), Peptide delivery, Drug delivery, Porphyromonas gingivalis, Streptococcus gordonii, Periodontal disease, Oral biofilm

\section{Background}

Periodontal disease is a group of chronic inflammatory diseases commonly caused by Porphyromonas gingivalis,

\footnotetext{
*Correspondence: drdemu01@louisville.edu; jmstei01@louisville.edu ${ }^{\dagger}$ Donald R. Demuth and Jill M. Steinbach-Rankins are Co-Senior authors ${ }^{1}$ Department of Oral Immunology and Infectious Diseases, University of Louisville School of Dentistry, 501 S. Preston St, Louisville, KY 40202, USA

2 Department of Bioengineering, University of Louisville Speed School of Engineering, 505 S. Hancock St., Room 623, Louisville, KY 40202, USA Full list of author information is available at the end of the article
}

Tannerella forsythia, and Treponema denticola. Together these pathogens are known as the "red complex" [1]. The progression of periodontal disease can cause tissue destruction and tooth loss, and if left untreated can contribute to systemic conditions of increased cancer risk, cardiovascular disease, diabetes, rheumatoid arthritis, pulmonary disease, and obesity $[2,3]$.

Current periodontal treatments aim to reduce bacterial plaque formation in the oral cavity using primarily physical and chemical (antibiotic) methods [4, 5]. However, current antibiotic treatment strategies exhibit 
non-specific activity, affecting beneficial organisms also present in the oral microbiome. Additional potential risks include the development of anti-bacterial resistant species, emergence of fungal opportunistic infections or Pseudomonas infection, and allergic reactions. Last, most current antibiotics have difficulty penetrating periodontal biofilms, and must be frequently administered, due to their transient activity in the oral cavity [6-8].

Porphyromonas gingivalis has been found to be associated with chronic periodontitis in $88 \%$ of sub-gingival plaque samples [9]. Moreover, P. gingivalis and S. gordonii association enhances the disruption of host-microbe homeostasis and induces population changes in the subgingival biofilm, driving inflammatory periodontal diseases [10-12]. Previous work in our group has shown that $P$. gingivalis adherence to streptococci is driven by the interaction of the minor fimbrial antigen (Mfa) of $P$. gingivalis and the streptococcal antigen I/II (AgI/II) [13, 14]. From these studies, a peptide (designated BAR), was developed that potently inhibits $P$. gingivalis/S. gordonii adherence in vitro and reduces $P$. gingivalis virulence in a mouse model of periodontitis [15-17]. While efficacious, one of the challenges to free BAR administration is that it provides relatively transient inhibition of $P$. gingivalis in the oral cavity. Moreover, to treat established biofilms, relative to initial biofilm formation, higher concentrations of BAR are required.

Polymeric delivery vehicles provide one option to address these challenges, by offering prolonged and targeted delivery of active agents. In particular, for application to the oral cavity, polymeric nanoparticles (NPs) are easy to fabricate and produce stable formulations. From a delivery perspective, polymeric NPs may offer rapid degradation in the acidic environment of the oral cavity, while providing mucoadhesive properties due to the electrostatic interactions between NPs and gingival epithelium [18-20]. Furthermore, for more labile molecules like biologics, polymers have the potential to protect the functionality of the active agent and provide tunable release and prolonged delivery, while enabling localization of the active agent to target sites [19, 21]. In addition polymeric NPs may offer a safer and more biocompatible delivery method, relative to currently applied metallic NPs that exhibit broad antimicrobial effect [22, 23].

Previous work in our groups has demonstrated that NPs surface-modified with BAR peptide more potently inhibit $P$. gingivalis adherence to $S$. gordonii, relative to an equimolar administration of free BAR peptide in vitro [24]. This increased potency was attributed to a higher localized dose of BAR, facilitating multivalent interactions with $P$. gingivalis. While surface-modified NPs provide targeting efficacy, a method of delivering high concentrations of BAR for prolonged duration has not been investigated. In this study, we sought to develop a formulation that encapsulates and prolongs the delivery of BAR, for durations relevant to oral delivery. BAR-encapsulated PLGA NPs were characterized and evaluated in two-species biofilm inhibition and disruption models. In addition, the kinetics of BAR-encapsulated, relative to BAR surfacemodified NPs were assessed in a two-species model.

\section{Methods \\ Peptide synthesis}

BAR peptide is comprised of residues 1167 to 1193 of the SspB (Antigen I/II) protein sequence of $S$. gordonii $\left(\mathrm{NH}_{2}\right.$-LEAAPKKVQDLLKKANITVKGAFQLFS -COOH) [16]. To enable peptide quantification and detection, the epsilon amine of the underlined lysine residue of BAR was covalently reacted with 6-carboxyfluorescein to produce fluorescent BAR (F-BAR). Both unlabeled and labeled peptides were synthesized by BioSynthesis, Inc. (Lewisville, TX) and obtained with greater than $90 \%$ purity.

\section{BAR-encapsulated and BAR surface-modified nanoparticle synthesis}

BAR and F-BAR encapsulated poly(lactic-co-glycolic acid) PLGA and methoxy-polyethylene glycol (mPEG-PLGA) NPs were synthesized using a double emulsion technique $[25,26]$. Briefly, BAR was encapsulated in PLGA carboxyl-terminated polymer $\left(0.55-0.75 \mathrm{dL} / \mathrm{g}\right.$; LACTEL ${ }^{\circledR}$; DURECT Corporation, Cupertino, CA, USA) or mPEGPLGA (Mw 5000:55,000 Da; PolySciTech ${ }^{\circledR}$; Akina, Inc., IN, USA). One hundred milligrams of PLGA or mPEGPLGA was dissolved in $2 \mathrm{~mL}$ methylene chloride (DCM) overnight. The next day, BAR was dissolved in $200 \mu \mathrm{L}$ Tris EDTA (TE) buffer at a concentration of $43 \mu \mathrm{g}$ BAR $/ \mathrm{mg}$ PLGA. The resulting PLGA/DCM solution was vortexed while adding $200 \mu \mathrm{L}$ of BAR peptide solution dropwise, and the mixture was ultrasonicated. Next, $2 \mathrm{~mL}$ of the PLGA/DCM/BAR solution was added dropwise to $2 \mathrm{~mL}$ of $5 \%(\mathrm{w} / \mathrm{v})$ polyvinyl alcohol (PVA) while vortexing and was subsequently sonicated. The NP solution was added to $50 \mathrm{~mL}$ of $0.3 \%$ PVA for $3 \mathrm{~h}$ to evaporate residual DCM. After evaporation, the NP solution was centrifuged at $13,000 \mathrm{rpm}$ at $4{ }^{\circ} \mathrm{C}$ and washed with distilled water twice. F-BAR encapsulated NPs were synthesized similarly, but were protected from light to avoid photobleaching.

BAR surface-modified NPs were synthesized similarly as above using a previously described double emulsion technique [26-29]. Briefly, the $5 \%(\mathrm{w} / \mathrm{v})$ polyvinyl alcohol (PVA) solution was mixed with $2 \mathrm{~mL}$ of $5 \mathrm{mg} / \mathrm{mL}$ avidinpalmitate and the $2 \mathrm{~mL}$ PLGA/DCM solution was added dropwise to $4 \mathrm{~mL} \mathrm{PVA} /$ avidin-palmitate while vortexing. After the first wash, the supernatant was discarded and the pelleted NPs were resuspended in $10 \mathrm{~mL}$ PBS for $30 \mathrm{~min}$ on a benchtop rotator, with biotinylated BAR 
peptide at a molar ratio of 3:1 BAR:avidin $(18.5 \mathrm{nmol} /$ $\mathrm{mg}$ ) in PBS. After conjugation, the NPs were washed two times with distilled water by centrifugation at 13,000 rpm at $4{ }^{\circ} \mathrm{C}$. After washing, both BAR-encapsulated and BAR surface-modified NPs, were suspended in $5 \mathrm{~mL}$ of distilled water, frozen at $-80^{\circ} \mathrm{C}$, and lyophilized.

\section{NP characterization: NP morphology, size, BAR loading, controlled release}

Unhydrated NP morphology, diameter, and size distribution were determined by analyzing scanning electron microscopy (SEM) images with NIH ImageJ software (version 1.5a, imageJ.nih.gov). Dynamic light scattering and zeta potential analyses were performed on hydrated NPs to determine the hydrodynamic diameter and surface charge (Malvern, Malvern, UK). To determine BAR loading and encapsulation efficiency (EE), NPs were dissolved in dimethyl sulfoxide (DMSO). The quantity of extracted F-BAR was determined by measuring fluorescence (488/518 nm excitation/emission). For BAR-encapsulated NPs, in vitro release was measured by gentle agitation of NPs in phosphate buffered saline (PBS, $\mathrm{pH}$ 7.4) at $37^{\circ} \mathrm{C}$. At fixed time points (1, 2, 4, 8, 24, $48 \mathrm{~h}$ ), samples were collected and the amount of BAR released from the NPs was quantified as described above.

\section{Growth of bacterial strains}

Porphyromonas gingivalis ATCC 33277 was grown in Trypticase soy broth (Difco Laboratories Inc., Livonia, MI, USA) supplemented with $0.5 \%(\mathrm{w} / \mathrm{v})$ yeast extract, $1 \mu \mathrm{g} / \mathrm{mL}$ menadione, and $5 \mu \mathrm{g} / \mathrm{mL}$ hemin. The medium was reduced for $24 \mathrm{~h}$ under anaerobic conditions $(10 \%$ $\mathrm{CO}_{2}, 10 \% \mathrm{H}_{2}$, and $80 \% \mathrm{~N}_{2}$ ) and $P$. gingivalis was subsequently inoculated and grown anaerobically for $48 \mathrm{~h}$ at $37{ }^{\circ} \mathrm{C}$. S. gordonii DL-1 was cultured aerobically without shaking in brain-heart infusion broth (Difco Laboratories Inc.) supplemented with $1 \%$ yeast extract for $16 \mathrm{~h}$ at $37^{\circ} \mathrm{C}$.

\section{Biofilm inhibition assay}

To assess the effectiveness of BAR-encapsulated NPs to prevent the interaction of $P$. gingivalis with $S$. gordonii, $S$. gordonii was harvested from culture and labeled with $20 \mu \mathrm{L}$ of $5 \mathrm{mg} / \mathrm{mL}$ hexidium iodide for $15 \mathrm{~min}$ at room temperature. Following incubation, cells were centrifuged to remove unbound fluorescent dye. Subsequently, the bacterial concentration was measured by the O.D. at $600 \mathrm{~nm}$ from 20 -fold diluted cultures of S. gordonii. The optical density of $S$. gordonii cells was adjusted to $0.8\left(1 \times 10^{9} \mathrm{CFU} / \mathrm{mL}\right)$ to obtain uniformity between cell counts in each well. After adjusting the optical density, $1 \mathrm{~mL}$ of $S$. gordonii cells was added to each well of 12-well culture plates containing a sterilized micro-coverslip. The cell culture plates were wrapped in aluminum foil to protect the labeled cells from light and placed on a rocker platform in the anaerobic chamber for $24 \mathrm{~h}$.

Porphyromonas gingivalis cultures were optimized using a similar approach, utilizing a different fluorescent label $(20 \mu \mathrm{L}$ of $4 \mathrm{mg} / \mathrm{mL}$ carboxyfluorescein-succinylester). $P$. gingivalis was incubated with the fluorescent dye for $30 \mathrm{~min}$ on a rocker platform and protected from light. The same procedures were followed as performed with $S$. gordonii to determine cell concentration, with slight adaptations. The optical density of $P$. gingivalis was adjusted from 0.8 to 0.4 O.D. $\left(5 \times 10^{7} \mathrm{CFU} / \mathrm{mL}\right)$ by diluting $P$. gingivalis cultures with an equal volume of BAR NPs or free BAR. The final concentration of BAR NPs or free BAR ranged from 0.3 to $3 \mu \mathrm{M}$ based on the previously determined IC50 of free BAR $(1.3 \mu \mathrm{M})$. P. gingivalis was incubated with BAR NPs or free BAR at $25{ }^{\circ} \mathrm{C}$ for 30 min before transferring to wells containing S. gordonii.

Plates containing $P$. gingivalis and $S$. gordonii were subsequently incubated for $24 \mathrm{~h}$ at $37^{\circ} \mathrm{C}$ in anaerobic conditions [24]. The following day, the supernatant was removed and cells were washed with PBS. Adherent cells were fixed with $4 \%(\mathrm{w} / \mathrm{v})$ paraformaldehyde and the cover glass was mounted on a glass slide. Biofilms were visualized using a Leica SP8 confocal microscope (Leica Microsystems Inc., Buffalo Grove, IL) under $60 \times$ magnification. Background noise was minimized using software provided with the Leica SP8 and three-dimensional z-stack biofilm images were obtained from 30 randomly chosen frames using a z-step size of $0.7 \mu \mathrm{m}$. Images were analyzed with Volocity image analysis software (version 6.3; Perkin Elmer, Waltham, MA, USA) to determine the ratio of green to red fluorescence (GR), representing P. gingivalis and S. gordonii, respectively. Control samples were used to subtract background levels of auto-fluorescence. Briefly, triplicate samples of $S$. gordonii alone were immobilized without P.g or BAR in 12-well culture plates and the same procedures for dual-species biofilm were followed. S. gordoniionly coverslips were visualized and images were analyzed using the previously mentioned approach. GR background was subtracted using the following formula: GR sample or control - GR S. gordonii-only. Each treatment group (BAR NPs or free BAR) was analyzed in triplicate and three independent frames were measured for each well. The mean and variation (SD) between samples were determined using analysis of variance (ANOVA) and differences were considered to be statistically significant when $\mathrm{p}<0.05$. The percent inhibition of $P$. gingivalis adherence was calculated with the following formula: GR sample/GR control.

\section{Biofilm disruption assay}

The same procedures utilized in the inhibition assay were followed, except $P$. gingivalis was allowed to adhere to 
streptococci in the absence of BAR peptide or BAR NPs to demonstrate the ability of BAR-encapsulated NPs to disrupt or "treat" pre-established biofilms. The resulting P. gingivalis/S. gordonii biofilms were then treated for $3 \mathrm{~h}$ with free BAR or BAR-encapsulated NPs at various concentrations and processed and analyzed as described above.

\section{Inhibitory kinetics of BAR released from BAR-encapsulated NPs}

Due to the similar release properties of BAR from PLGA and mPEG-PLGA NPs, PLGA NPs were selected to further assess the ability of NPs to release therapeutically relevant concentrations of BAR at different time points. PLGA BAR NPs $(1.3 \mu \mathrm{M})$ were incubated with gentle agitation in PBS (pH 7.4) at $37^{\circ} \mathrm{C}$. After 1, 2, 4 and $8 \mathrm{~h}$, the NP suspension was centrifuged, and the supernatant was collected for biofilm experiments. The NPs were re-suspended with new PBS. P. gingivalis was incubated with BAR NP eluate for $30 \mathrm{~min}$, and subsequently transferred to a well containing an $S$. gordonii biofilm. The same biofilm inhibition assay procedure detailed above was used to visualize and analyze the samples.

\section{Time-dependent comparison between free BAR,} BAR-encapsulated, and BAR surface-modified NPs

In addition to delivering high concentrations of BAR during the time frame of interest, the temporal evaluation of BAR activity against established biofilms was evaluated and compared. Both BAR-encapsulated and BAR surfacemodified NPs were assessed due to their previously demonstrated efficacy. P. gingivalis was allowed to adhere to streptococci in the absence of peptide, then BAR $(3 \mu \mathrm{M})$, BAR-encapsulated, and BAR surface-modified NPs (1.3 and $3 \mu \mathrm{M})$ were applied to the biofilms. The biofilms were assessed 1, 2, and $3 \mathrm{~h}$ post-administration and visualized as described above.

\section{Results}

Nanoparticle characterization

The morphology, size, and zeta potential of BAR PLGA and mPEG-PLGA NPs were determined. The morphologies of BAR-encapsulated PLGA and mPEG-PLGA NPs are shown in Fig. 1. Both PLGA and mPEG-PLGA NPs demonstrated spherical morphology with average unhydrated diameters of $227.5 \pm 23.0 \mathrm{~nm}$ and $243.1 \pm 31.2 \mathrm{~nm}$ respectively (Table 1). In comparison, the average hydrated diameters of PLGA and mPEG-PLGA NPs were $234.4 \pm 19.2 \mathrm{~nm}$ and $278.9 \pm 13.8 \mathrm{~nm}$, respectively. PLGA and mPEG-PLGA NPs had zeta potentials of $-13.1 \pm 0.4 \mathrm{mV}$ and $-5.9 \pm 0.1 \mathrm{mV}$.

\section{Quantification of BAR loading and release}

The loading of BAR peptide in PLGA and mPEG-PLGA NPs was determined using fluorescence spectroscopy, and the fluorescence was compared to a known standard of F-BAR. Loading experiments demonstrated that both PLGA and mPEG-PLGA NPs highly encapsulated BAR with $19.0 \pm 0.1$ and $16.1 \pm 0.2 \mu \mathrm{g}$ of BAR per mg of NP,

\section{Table 1 Physical characterization of NPs}

\begin{tabular}{llll}
\hline NP type & $\begin{array}{l}\text { Unhydrated } \\
\text { diameter } \\
(\mathbf{n m})\end{array}$ & $\begin{array}{l}\text { Hydrated } \\
\text { diameter } \\
(\mathbf{n m})\end{array}$ & Zeta potential $(\mathbf{m V})$ \\
\hline PLGA NPS & $227.5 \pm 23.0$ & $234.4 \pm 19.2$ & $-13.1 \pm 0.4$ \\
mPEG-PLGA NPS & $243.1 \pm 31.2$ & $278.9 \pm 13.8$ & $-5.9 \pm 0.1$
\end{tabular}
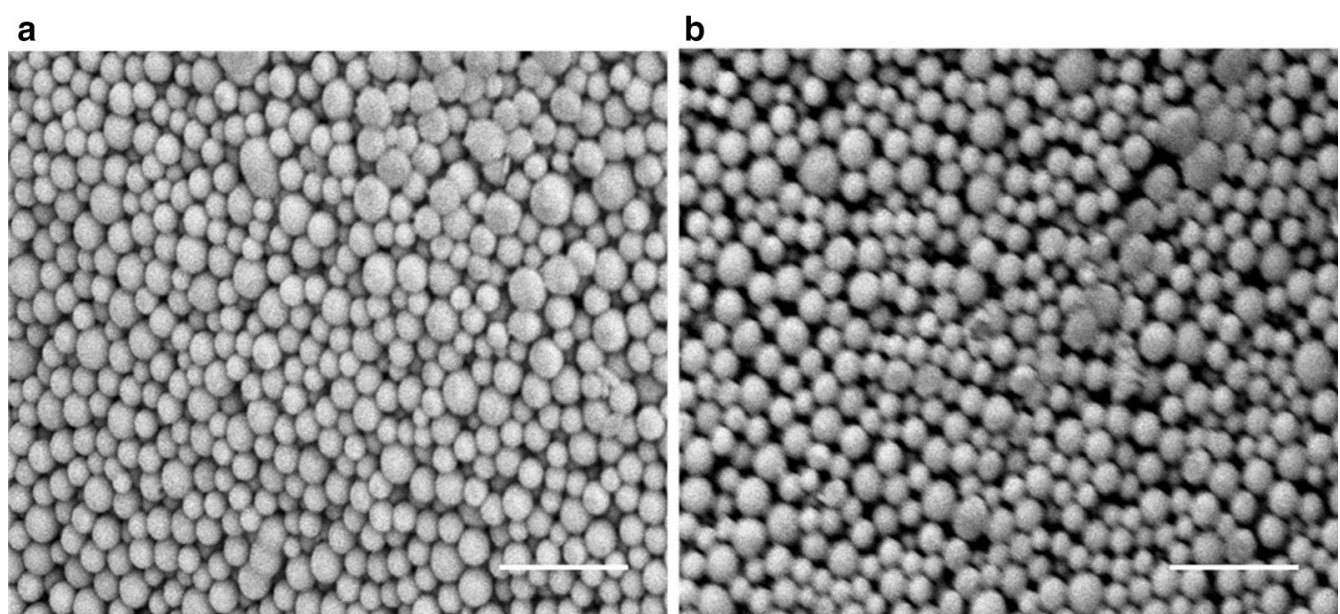

Fig. 1 SEM images of BAR-encapsulated a PLGA NPs and $\mathbf{b}$ mPEG-PLGA NPs. Scale bars represent $1 \mu \mathrm{m}$ 
Table 2 The amount of BAR $(\mu \mathrm{g})$ loaded in PLGA and $\mathrm{mPEG}$-PLGA NPs (mg)

\begin{tabular}{llll}
\hline NP type & $\begin{array}{l}\text { BAR input } \\
(\boldsymbol{\mu} \mathbf{g} / \mathbf{m g})\end{array}$ & BAR output $(\boldsymbol{\mu g} / \mathbf{m g})$ & $\begin{array}{l}\text { Encapsulation } \\
\text { efficiency }(\%)\end{array}$ \\
\hline PLGA NPS & 43 & $19.0 \pm 0.1$ & 44.2 \\
mPEG-PLGA NPS & 43 & $16.1 \pm 0.2$ & 37.3 \\
\hline
\end{tabular}

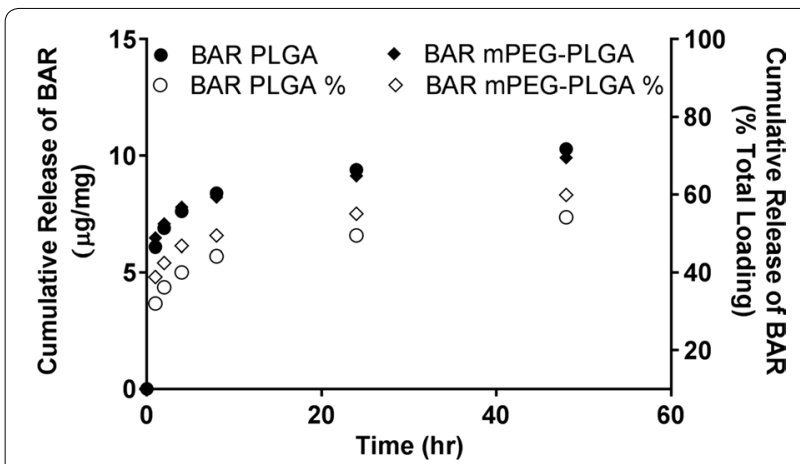

Fig. 2 Cumulative release of BAR as a function of mass ( $\mu \mathrm{g} B A R$ per mg NP, open symbols) and percent of total BAR loaded (closed symbols) over $48 \mathrm{~h}$ respectively, corresponding to encapsulation efficiencies of 44 and $37 \%$ (Table 2).

To assess BAR release from the NPs, the fluorescence of supernatant from 1, 2, 4, 8, 24, 48 h release time points was measured and compared to a known standard of F-BAR in PBS. Release experiments demonstrated that $47 \%$ of encapsulated BAR $(10.3 \mu \mathrm{g} / \mathrm{mg})$ was released from PLGA NPs, while $56 \%$ of BAR $(9.9 \mu \mathrm{g} / \mathrm{mg})$ was released from mPEG-PLGA NPs within $24 \mathrm{~h}$ (Fig. 2).

Inhibition (or prevention) of $P$. gingivalis/S. gordonii biofilm formation

BAR-encapsulated PLGA and mPEG-PLGA NPs were functionally evaluated to determine their potential to inhibit $P$. gingivalis adherence to $S$. gordonii after $24 \mathrm{~h}$, relative to free BAR. As shown in Fig. 3 and Additional file $1, P$. gingivalis adherence was significantly reduced in the presence of BAR-encapsulated PLGA and mPEGPLGA NPs. Adherence was inhibited by $39 \%$ at the lowest administered concentration $(0.3 \mu \mathrm{M}), 59 \%$ at $0.7 \mu \mathrm{M}$, and reached maximum inhibition (94\%) at the highest concentration of PLGA NPs tested $(3 \mu \mathrm{M})$. Similar inhibitory results were observed for mPEG-PLGA

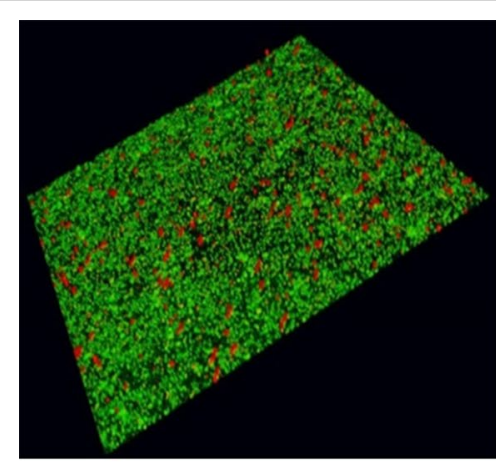

Control

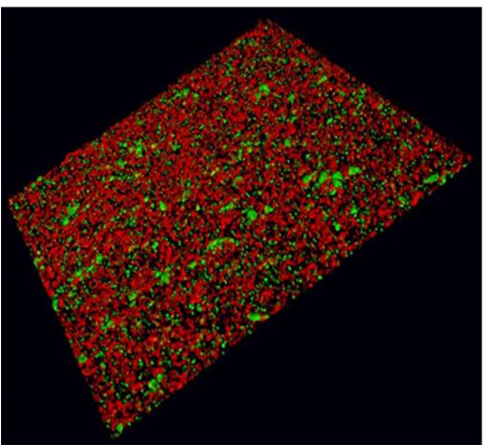

1.3 $\mu$ M BAR-encap PLGA

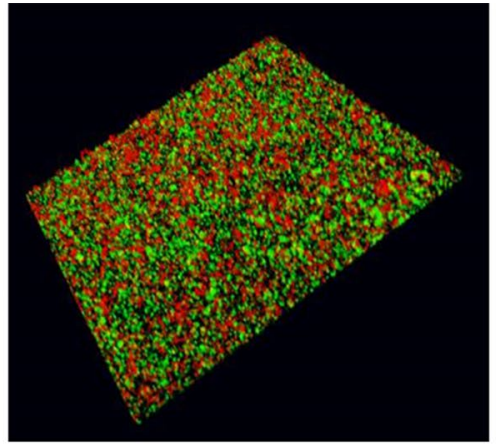

$0.3 \mu$ M BAR-encap PLGA

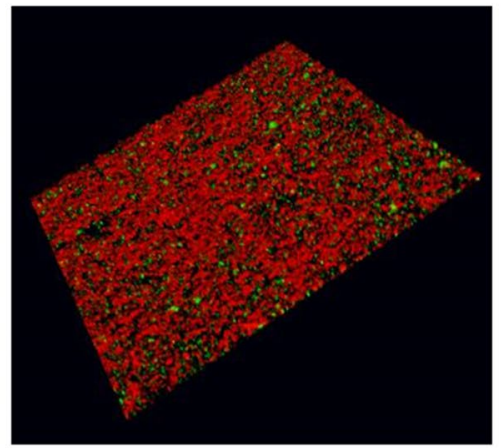

2 MM BAR-encap PLGA

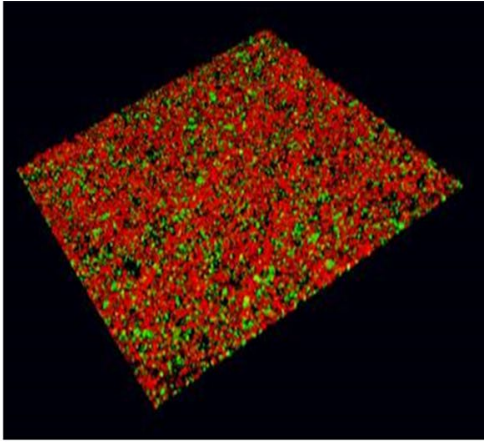

$0.7 \mu \mathrm{M}$ BAR-encap PLGA

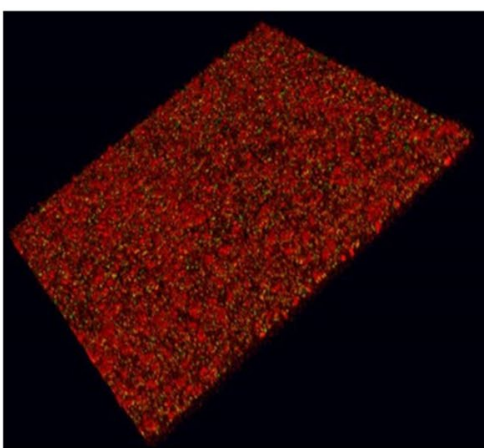

$3 \mu \mathrm{M}$ BAR-encap PLGA

Fig. 3 BAR-encapsulated PLGA NPs prevent P. gingivalis adherence to S. gordonii. Biofilms were visualized with confocal microscopy and the ratio of green (P. gingivalis) to red (S. gordonii) fluorescence in $z$-stack images was determined using Volocity image analysis software. Each grid represents $21 \mu \mathrm{m}$ 
NPs, where P.g/S.g biofilm formation was inhibited by 37,55 , and $92 \%$ at concentrations of $0.3 \mu \mathrm{M}, 0.7 \mu \mathrm{M}$ and $3 \mu \mathrm{M}$ respectively. The ability of BAR-encapsulated NPs to inhibit biofilm formation was dose-dependent $(\mathrm{IC} 50=0.7 \mu \mathrm{M})$ with no statistically significant differences between PGLA and mPEG-PLGA BAR-encapsulated NPs $(p>0.05)$. Moreover these results indicate that a lower concentration of BAR is required if incorporated within NPs, relative to free BAR administration $(\mathrm{IC} 50=1.3 \mu \mathrm{M})$ (Figs. 3 and $5 \mathrm{a})$.

\section{Disruption (or treatment) of $P$. gingivalis/S. gordonii biofilms}

To determine whether BAR peptide is capable of disrupting pre-existing $P$. gingivalis/S. gordonii biofilms, dual-species biofilms were formed in PBS in the absence of BAR peptide for $24 \mathrm{~h}$, and were subsequently incubated for $3 \mathrm{~h}$ with BAR-encapsulated PLGA or mPEGPLGA NPs. Various molar concentrations of BAR NPs ranging from 0.3 to $3 \mu \mathrm{M}$ were tested. The biofilms were visualized and the percent inhibition was calculated as described above. As shown in Fig. 4 and Additional file 2, BAR-encapsulated PLGA and mPEG-PLGA NPs disrupted pre-existing dual-species biofilms by $\sim 25 \%$ with the lowest administered concentration $(0.3 \mu \mathrm{M})$, $40 \%$ with $0.7 \mu \mathrm{M}$, and $85 \%$ with $3 \mu \mathrm{M}$ of BAR-encapsulated PLGA NPs. Similar trends were observed for the disruption of pre-existing biofilms with $0.3,0.7$, and $3 \mu \mathrm{M}$ mPEG-PLGA NPs (20\%, 38\%, and 80\% disrupted). Overall the IC50 values of PLGA and mPEG-PLGA $(\sim 1.3 \mu \mathrm{M})$ NPs for biofilm disruption were not statistically different ( $p>0.05$, Fig. $5 b$ ); demonstrating statistically significant improvements in efficacy relative to free $\operatorname{BAR}(\mathrm{p}<0.05)$.

\section{Inhibitory activity of BAR released from BAR-encapsulated} NPs

To determine the inhibitory potential of BAR-encapsulated NPs, as a function of release duration, streptococcal cells were immobilized and $P$. gingivalis was incubated with eluate released from $1.3 \mu \mathrm{M}$ BARencapsulated PLGA NPs at 1, 2, 4, and 8 h. BAR-encapsulated PLGA NPs were selected due to their similar release and inhibitory properties, relative to $\mathrm{MPEG}$ PLGA NPs. As shown in Fig. 6, BAR released during the first $2 \mathrm{~h}$, potently inhibited biofilm formation $(68 \%$

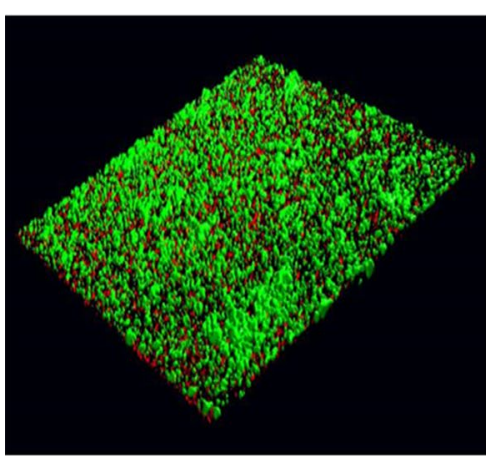

Control

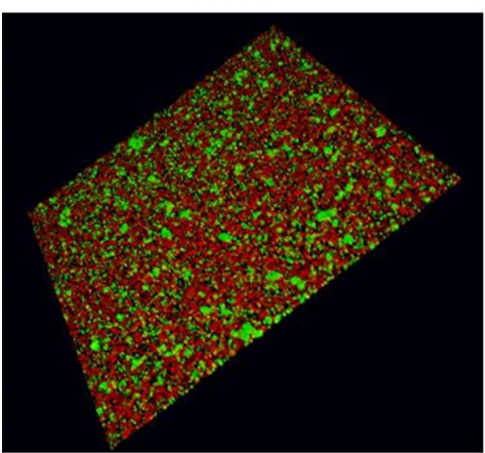

1.3 $\boldsymbol{\mu M}$ BAR-encap PLGA

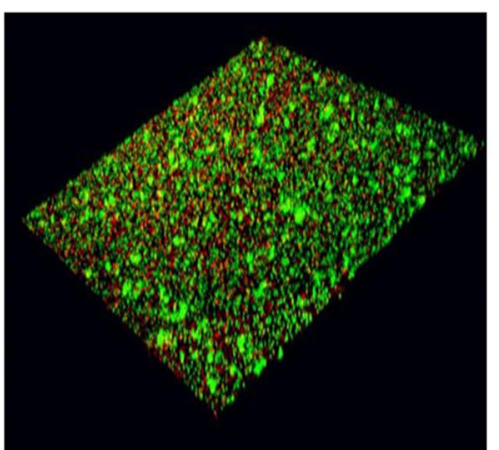

$0.3 \mu \mathrm{M}$ BAR-encap PLGA

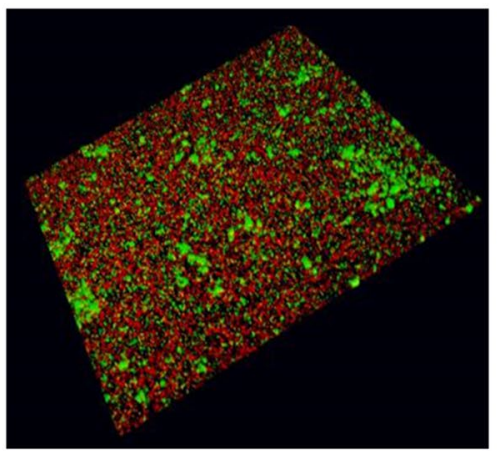

2 $\mu$ M BAR-encap PLGA

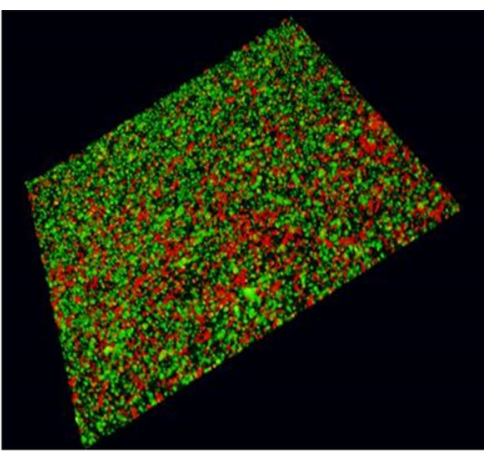

$0.7 \mu M$ BAR-encap PLGA

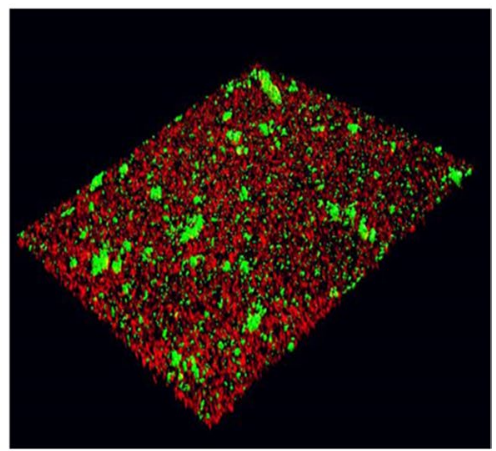

3 MM BAR-encap PLGA

Fig. 4 BAR-encapsulated PLGA NPs disrupt pre-established P. gingivalis-S. gordonii biofilms. Biofilms were visualized with confocal microscopy and the ratio of green (P. gingivalis) to red (S. gordonii) fluorescence in z-stack images was determined using Volocity image analysis software. Each grid represents $21 \mu \mathrm{m}$ 

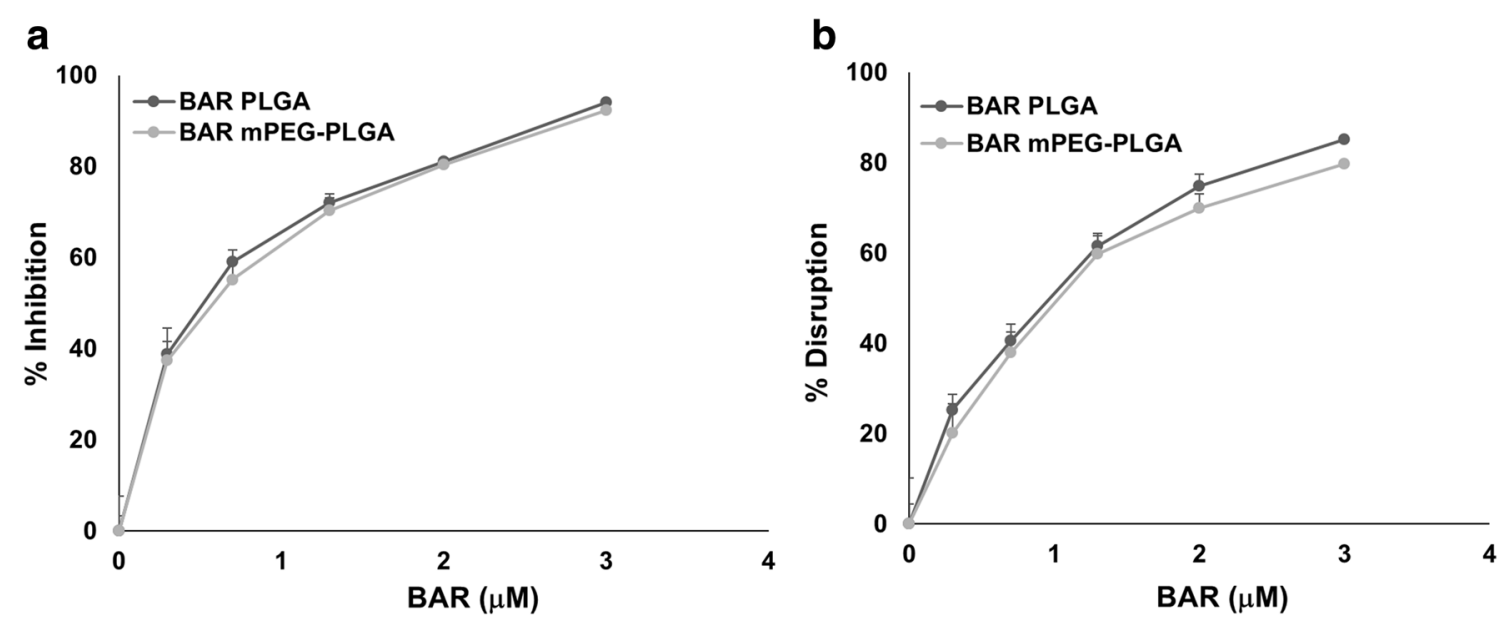

Fig. 5 Comparison of the concentration of BAR-encapsulated PLGA and mPEG-PLGA NPs needed to a inhibit or $\mathbf{b}$ disrupt P. gingivalis/S. gordonii biofilms

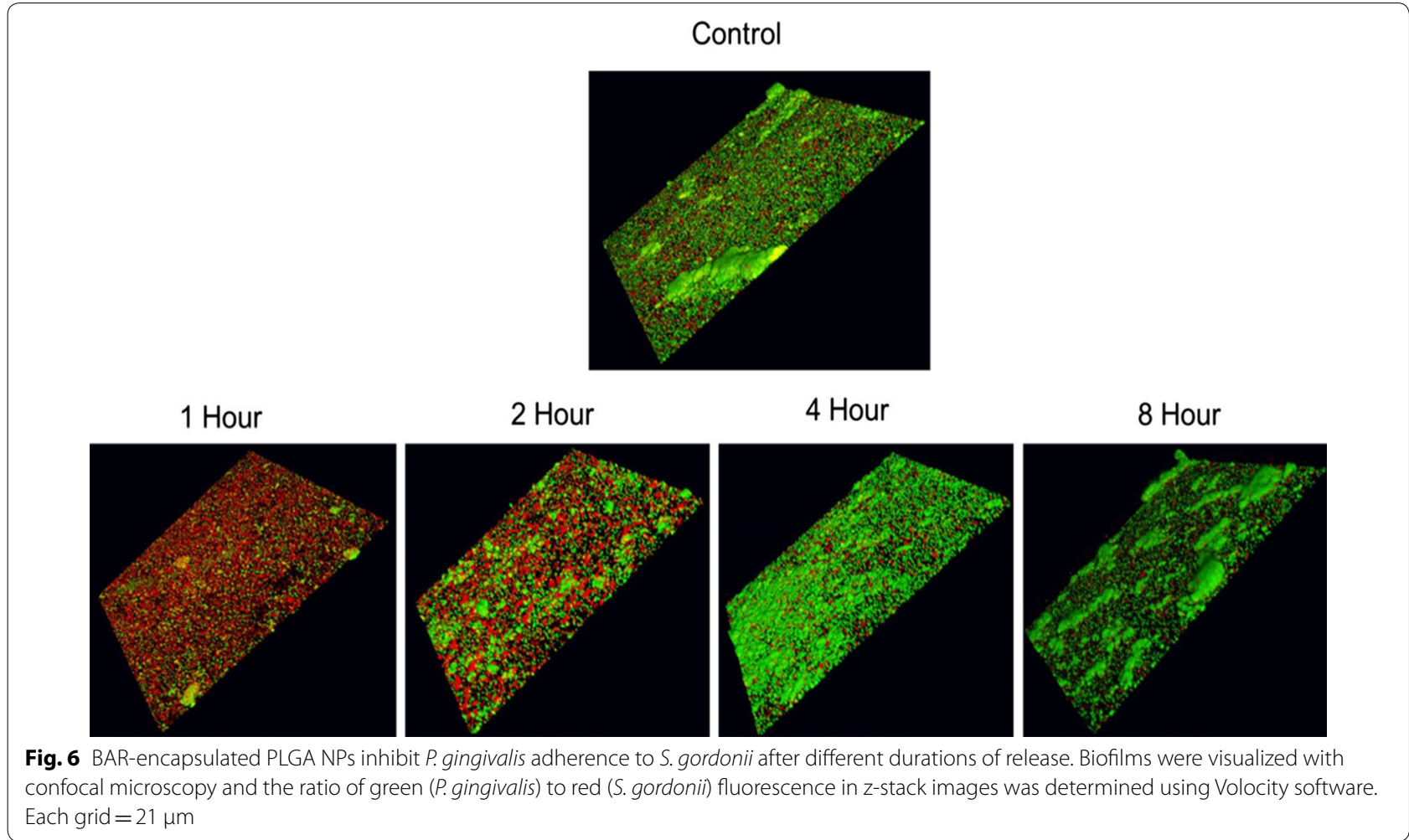

and $32 \%$, respectively), whereas BAR released after 4 and $8 \mathrm{~h}$ provided less potent inhibition of biofilm formation ( $25 \%$ for both time points). These results indicate that BAR-encapsulated NPs release an inhibitory dose of peptide for at least $2 \mathrm{~h}$.
Time-dependent comparison of free BAR,

BAR-encapsulated, and BAR surface-modified NP biofilm disruption

Previous studies demonstrated that BAR surfacemodified PLGA NPs potently disrupt pre-established 
P. gingivalis/S. gordonii biofilms [24]. To compare the temporal effect resulting from the administration of the newly formulated BAR-encapsulated NPs, relative to free BAR or previously tested BAR surface-modified NPs, two concentrations of BAR-encapsulated and BAR surfacemodified PLGA NPs were compared with free BAR after 1,2 , and $3 \mathrm{~h}$ administration to pre-established biofilms. As shown in Table 3 and Fig. 7, free BAR $(3 \mu \mathrm{M})$ minimally disrupted pre-existing biofilms during the first hour of application (23\%), and demonstrated a slight increase in disruption after $2 \mathrm{~h}(44 \%)$. After $3 \mathrm{~h}$, free BAR $(3 \mu \mathrm{M})$ disrupted $69 \%$ of the pre-existing biofilm. In comparison, administration of the same equimolar concentration of BAR-encapsulated NPs $(1.3$ and $3 \mu \mathrm{M})$ disrupted the established biofilm during the first hour of exposure by $32 \%$ and $38 \%$, respectively and demonstrated even more potent disruption (47\% and 52\%) after $2 \mathrm{~h}$. The maximum disruption for 1.3 and $3 \mu \mathrm{M}$ doses $(66 \%$ and $77 \%$, respectively) was achieved after $3 \mathrm{~h}$ exposure to biofilms. Comparatively, both 1.3 and $3 \mu \mathrm{M}$ BAR surfacemodified NPs disrupted pre-existing biofilms within $1 \mathrm{~h}$ by $43 \%$ and $49 \%$, respectively, and induced more potent biofilm disruption (59\% and 69\%) after $2 \mathrm{~h}$ exposure, demonstrating statistically significant disruption, relative to disruption induced by free BAR peptide. The highest levels of disruption ( $71 \%$ and $83 \%$ respectively) were achieved after $3 \mathrm{~h}$ BAR surface-modified NP administration. Overall, BAR surface-modified NPs were statistically more effective than free BAR $(\mathrm{p}<0.05)$ in disrupting established biofilms after 1,2 , and $3 \mathrm{~h}$ administration. However, no statistical differences were observed for BAR-encapsulated NPs ( $p>0.05)$, relative to BAR surface-modified NPs or free BAR peptide after 1, 2, or $3 \mathrm{~h}$ administrations.

\section{Discussion}

Porphyromonas gingivalis has been identified as a "keystone" pathogen involved in the initiation and progression of periodontal inflammatory disease, by disrupting host-microbe homeostasis and inducing population changes in the subgingival biofilm. This disruption and colonization is initially prompted by the association of $P$. gingivalis with oral streptococci in the supragingival niche, and is thus an ideal target for therapeutic intervention [5]. Previous studies have shown that BAR peptide inhibits biofilm formation by $P$. gingivalis and $S$. gordonii in vitro and reduces the virulence of $P$. gingivalis in a murine model of infection [15-17]. While efficacious, BAR effectiveness was limited by the duration of exposure within the oral cavity, and necessitated a higher concentration to disrupt previously established biofilms [15-17]. In previous work we sought to address these challenges by synthesizing BAR surface-modified NPs to multivalently inhibit biofilm formation [24]. The goal of this study was to develop, characterize, and compare BAR-encapsulated NPs that release BAR within a time frame relevant to delivery in the oral cavity.

Nanoparticle characterization revealed that PLGA and mPEG-PLGA BAR-encapsulated NPs exhibited spherical morphologies and average particle diameters of $234.4 \pm 19.2 \mathrm{~nm}$ and $278.9 \pm 13.8 \mathrm{~nm}$, with respective zeta potentials of $-13.1 \pm 0.4 \mathrm{mV}$ and $-5.9 \pm 0.1 \mathrm{mV}$. These values are in agreement with expected values for these polymeric NPs [24-26, 28, 29]. Both PLGA and mPEGPLGA NPs were synthesized with $43 \mu \mathrm{g}$ of BAR per mg NP, corresponding to loading concentrations deemed feasible for biofilm inhibition with free BAR [15-17]. PLGA and mPEG-PLGA NPs demonstrated relatively high peptide loading with $19.0 \pm 0.1$ and $16.1 \pm 0.2 \mu \mathrm{g}$ BAR per mg of NP respectively.

In addition to high loading, PLGA and mPEG-PLGA NPs released $40 \%$ and $48 \%$ of BAR within the first $4 \mathrm{~h}$, with no statistically significant differences between release profiles. The NP formulations were designed to achieve therapeutic concentrations of BAR in the oral cavity for a minimum of $2 \mathrm{~h}$. This initial window of $2 \mathrm{~h}$ release was targeted as we envision formulating NPs in a mouth rinse or toothpaste product. Ideally, in future formulations, we seek to tailor the release of peptide for up to $12 \mathrm{~h}$ since we envision these formulations may be applied once or twice daily, to exert immediate effect over a number of hours.

To assess the functionality of BAR-NPs, the inhibition and disruption concentrations of BAR-encapsulated PLGA and mPEG-PLGA NPs were determined against dual-species biofilms. As shown in Figs. 3 and 4, BAR NPs demonstrated potent inhibition and disruption with

Table 3 Percent disruption of pre-existing biofilms with different treatment groups and concentrations

\begin{tabular}{|c|c|c|c|c|c|}
\hline \multirow[t]{2}{*}{ Time (h) } & \multicolumn{5}{|c|}{ \% Disruption of pre-formed biofilms } \\
\hline & Free BAR $(3 \mu \mathrm{M})$ & $\begin{array}{l}\text { BAR-mod NPs } \\
(1.3 \mu \mathrm{M})\end{array}$ & BAR-mod NPs $(3 \mu \mathrm{M})$ & $\begin{array}{l}\text { BAR-encap NPs } \\
(1.3 \mu \mathrm{M})\end{array}$ & $\begin{array}{l}\text { BAR-encap } \\
\text { NPs }(3 \mu \mathrm{M})\end{array}$ \\
\hline 1 & $22.6 \pm 0.2$ & $43.4 \pm 0.2$ & $48.9 \pm 0.1$ & $32.3 \pm 0.1$ & $37.7 \pm 0.1$ \\
\hline 2 & $44.4 \pm 0.2$ & $59.2 \pm 0.1$ & $68.7 \pm 0.1$ & $46.6 \pm 0.2$ & $52.4 \pm 0.2$ \\
\hline 3 & $69.0 \pm 0.0$ & $71.2 \pm 0.1$ & $83.4 \pm 0.0$ & $66.1 \pm 0.1$ & $77.0 \pm 0.0$ \\
\hline
\end{tabular}




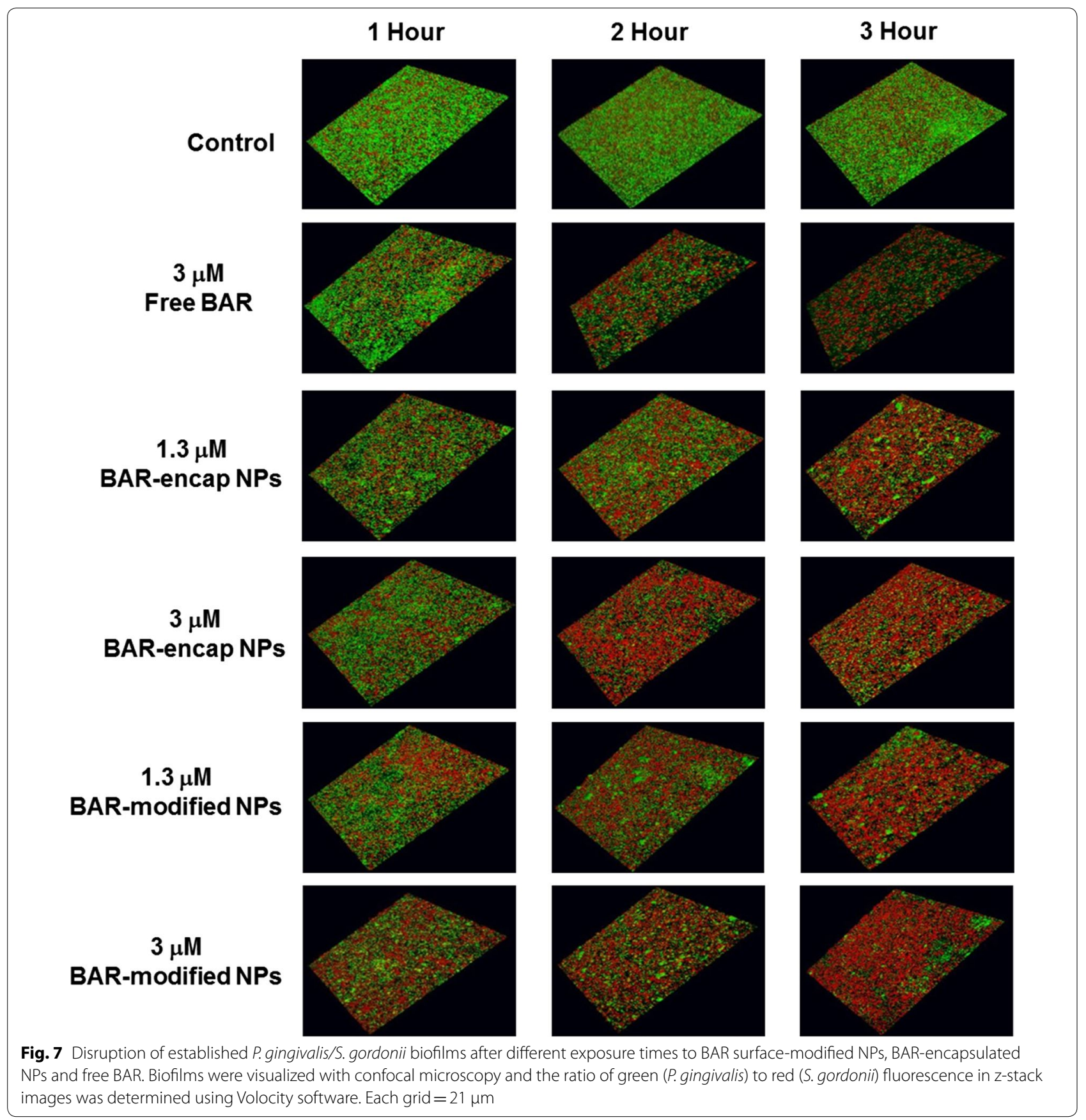

IC50 $\mathrm{s}=0.7 \mu \mathrm{M}$ and $1.3 \mu \mathrm{M}$, respectively, with negligible differences observed between PLGA and mPEGPLGA NPs (Additional file 1 and Additional file 2). To explore the temporal effect of BAR released from PLGA NPs on biofilm inhibition (prevention) in greater depth, the efficacy of BAR-encapsulated NPs was assessed in a dual-species biofilm after 1, 2, 4 and $8 \mathrm{~h}$ post-application. Sufficient BAR release was achieved, relating to inhibitory concentrations of $1.3 \mu \mathrm{M}$ during the first
$4 \mathrm{~h}$ of administration (Fig. 6). Moreover, the temporal dependence of free BAR, BAR-encapsulated, and BAR surface-modified NPs to disrupt pre-established biofilms (treatment) was measured after 1, 2, and $3 \mathrm{~h}$ application. As shown in Fig. 7 and Table 3, BAR-encapsulated and BAR surface-modified NPs achieved moderate biofilm inhibition within $1 \mathrm{~h}$ in a dose-dependent manner; however, similar concentrations of free BAR required prolonged exposure of up to $3 \mathrm{~h}$ to achieve more potent 
effect. These results demonstrate that BAR-encapsulated NPs provide a feasible alternative to free BAR and BAR surface-modified NPs to target dual-species oral biofilms and provide rapid onset of action. Together, these studies indicate that BAR-encapsulated NPs may serve as a short-term delivery formulation to enhance BAR delivery and potency in the oral cavity. Moreover, by encapsulating versus surface-modifying NPs with BAR, these NPs may offer the potential to specifically target NPs with modifications that can complement BAR activity to engage with these or other bacterial species in future work.

To date, a variety of polymeric nanoparticle formulations have been developed for oral delivery; however, these vehicles have primarily focused on the delivery of non-specific active agents such as antibiotics [30-39]. Antibiotics such as chlorhexidine [30,31], minocycline [32, 33], clarithromycin [36], vancomycin [34], doxycycline [37], and tetracycline [35, 38, 39] are among the antibiotics that have been incorporated into a variety of polymeric vehicles [30-37] to provide sustained-delivery, prolong activity, exert antibacterial activity, and decrease antibiotic cytotoxicity [30-37]. Yet, despite antibiotic choice, primary concerns of antibacterial resistance and cytotoxicity remain [30,31, 35]. While chitosan and PLGA NPs that encapsulated chlorhexidine dihydrochloride (CHX) demonstrated strong adherence to tooth surfaces and sustained-release for $48 \mathrm{~h}$ in neutral $\mathrm{pH}$ conditions, moderate cytotoxicity due to $\mathrm{CHX}$ was observed in human gingival fibroblasts [31]. Similar studies seeking to ameliorate periodontal infection caused by $A$. actinomycetemcomitans and $P$. nigrescens with PLGA lovastatin-chitosan-tetracycline NPs demonstrated potent inhibition up to 1 week after administration. However, significantly elevated alkaline phosphatase was observed in cells treated with $0.1 \%$ or $0.3 \%$ tetracycline-loaded nanoparticles on days 7 and 9 [35]. Overall, these studies have shown that delivery vehicles have the potential to increase antibiotic effectiveness by decreasing the concentration required. However, bacterial resistance, nonspecific targeting, and cytotoxicity concerns with chronic use suggest that the development of more specifically acting active agents will offer safer alternatives for biofilm inhibition.

More recently, specifically targeted biological agents have been investigated to treat periodontal diseases. Delivery of thyA gene [40], Punica granatum extract [41], H. madagascariensis leaf extract [42], miR-146a [43], and the anti-inflammatory agent 15d-PGJ2 [44] have been investigated to vaccinate against and target periodontal diseases. Recent work assessed the delivery of an oral vaccine comprised of an auxotrophic complementation of the thy $A$ gene to produce an immune response against S. gordonii. Although this study demonstrated promise utilizing $S$. gordonii as a live oral vaccine, to date there are few formulations available to localize or sustain biologic administration to the oral cavity [40]. In other work, PLGA NPs encapsulating a novel antiinflammatory agent (15d-PGJ2), demonstrated promise in reducing inflammatory response and bone resorption in mouse model of periodontitis after daily administration [44], demonstrating the feasibility of combined biologic and delivery vehicle against oral pathogens. Despite this recent progress in the delivery of biological agents for oral applications, currently few biological agents in combination with delivery vehicles have been developed to inhibit keystone-specific interactions during the initial stages of periodontal disease [24].

In addition to progress in the development of vehicles to encapsulate antibiotic and biological agents in polymeric delivery vehicles, polymeric platforms have also been surface-modified with a variety of molecules including RGD [33], chitosan [31, 36], tertiary amines bearing two t-cinnamaldehyde substituents [45], dimethyl-octyl ammonium [45], and BAR peptide [24] to increase the mucoadhesivity (and in the latter case, specificity) of oral delivery formulations. A variety of polymers have been modified with biological ligands to impart enhanced therapeutic effect $[24,33]$. As one example, the delivery of antibiotic minocycline-loaded poly(ethylene glycol)-poly(lactic acid) (PEG-PLA) nanoparticles have targeted oral epithelial cells by surface-modification with RGD peptides. Surface-modification of PEG-PLA NPs increased epithelial cell attachment and maintained effective drug concentrations in gingival fluid for more than 2 weeks in vivo, relative to unmodified minocycline NPs. Similarly, chitosan-modified polyvinyl caprolactampolyvinyl acetate-polyethylene glycol graft copolymer (Soluplus) and poly-(DL-lactide-co-glycolide) nanoparticles loaded with clarithromycin, increased antibacterial efficacy and provided sustained-release against oral biofilms [36]. Although this study demonstrated effective treatment of periodontitis, the limitations of antibiotic delivery still pose challenges [33]. Surface modification of nanoparticles has imparted new attributes to target active agents to oral-specific niches. We expect that combining our current work, with surface functionalization demonstrated in our previous study [24], may confer additional advantages in targeting keystone species by providing prevention and treatment via adhesion and a localized release-mediated platform.

Taken together, our results demonstrate that BARencapsulated NPs achieve more potent in inhibition and disruption than equimolar free BAR administration. We believe that incorporation of BAR peptide in NPs provides gradual release of BAR peptide, while 
BAR-modification offers a platform to provide a higher localized concentration of BAR in the oral cavity via multivalent interactions. BAR-encapsulated NPs offer a platform to improve efficacy, and potentially longevity in the oral cavity compared to the transient activity of free BAR. These experimental results will be helpful in developing NPs in therapeutic formulations such as toothpaste, mouth rinse or chewing gum. Future studies may focus on developing blended polymeric NPs to more gradually release inhibitory concentrations for $8-12 \mathrm{~h}$. Moreover, combining this platform with surface functionality to provide mucoadhesive or specific interactions with gingival tissue may be pursued to enhance the targeting potential. Ongoing and future work in our groups seeks to assess the efficacy of both BAR-modified and BAR-encapsulated NPs in a murine model of periodontitis.

\section{Additional files}

Additional file 1. BAR-encapsulated mPEG-PLGA NPs prevent $P$. gingivalis adherence to S. gordonii. Biofilms were visualized with confocal microscopy and the ratio of green (P. gingivalis) to red (S. gordonii) fluorescence in z-stack images was determined using Volocity image analysis software. Each grid $=21 \mu \mathrm{m}$.

Additional file 2. BAR-encapsulated mPEG-PLGA NPs disrupt preestablished $P$. gingivalis-S. gordonii biofilms. Biofilms were visualized with confocal microscopy and the ratio of green (P. gingivalis) to red (S.gordonii) fluorescence in z-stack images was determined using Volocity image analysis software. Each grid $=21 \mu \mathrm{m}$

\section{Authors' contributions}

MYM performed the experiments, statistical analysis, and drafted the manuscript. JMSR conceived of the study, participated in its design and coordination, and drafted the manuscript. DRD conceived of the study, participated in its design and coordination, and drafted the manuscript. All authors read and approved the final manuscript.

\section{Author details}

${ }^{1}$ Department of Oral Immunology and Infectious Diseases, University of Louisville School of Dentistry, 501 S. Preston St, Louisville, KY 40202, USA. 2 Department of Bioengineering, University of Louisville Speed School of Engineering, 505 S. Hancock St., Room 623, Louisville, KY 40202, USA. ${ }^{3}$ Department of Microbiology and Immunology, University of Louisville School of Medicine, Louisville, KY 40202, USA. ${ }^{4}$ Department of Pharmacology and Toxicology, University of Louisville School of Medicine, Louisville, KY 40202, USA. ${ }^{5}$ Center for Predictive Medicine, University of Louisville, 505 S. Hancock St, Louisville, KY 40202, USA.

\section{Acknowledgements}

This work was supported by grants R01DE023206 and R21DE025345 from the National Institute for Dental and Craniofacial Research. The authors declare no conflicts of interest. We will freely provide reagents and/or data generated in this study to academic investigators according to the policies of the University of Louisville. An abstract comprised of parts of this study was presented on March 24, 2017 at the International Association of Dental Research Meeting in San Francisco, CA.

\section{Competing interests}

The authors declare they have no competing interests.

\section{Availability of data and materials}

The datasets used and/or analyzed during the current study are available from the corresponding author on reasonable request. All data generated or analyzed during this study are included in this published article and its additional information files.

\section{Consent for publication \\ Not applicable.}

Ethics approval and consent to participate

Not applicable.

\section{Funding}

We gratefully acknowledge support from grants R01DE023206 (DRD) and R21DE025345 (DRD and JMSR) from the National Institute for Dental and Craniofacial Research.

\section{Publisher's Note}

Springer Nature remains neutral with regard to jurisdictional claims in published maps and institutional affiliations.

Received: 26 April 2018 Accepted: 5 September 2018

Published online: 15 September 2018

\section{References}

1. Hussain M, Stover CM, Dupont AP. gingivalis in periodontal disease and atherosclerosis - scenes of action for antimicrobial peptides and complement. Front Immunol. 2015;6:45.

2. Genco RJ, Van Dyke TE. Prevention: reducing the risk of CVD in patients with periodontitis. Nat Rev Cardiol. 2010;7(9):479-80.

3. Lundberg K, Wegner N, Yucel-Lindberg T, Venables PJ. Periodontitis in RA-the citrullinated enolase connection. Nat Rev Rheumatol. 2010;6(12):727-30

4. Ellen RP. Periodontal care for community-dwelling older adults. J Prosthet Dent. 1994;72(5):500-6.

5. KaldahI WB, Kalkwarf KL, Patil KD, Molvar MP, Dyer JK. Long-term evaluation of periodontal therapy: I. Response to 4 therapeutic modalities. J Periodontol. 1996;67(2):93-102.

6. Drisko CH. Non-surgical pocket therapy: pharmacotherapeutics. Ann Periodontol. 1996;1(1):491-566.

7. Walker CB. The acquisition of antibiotic resistance in the periodontal microflora. Periodontology. 1996;2000(10):79-88.

8. Allaker RP, lan Douglas CW. Non-conventional therapeutics for oral infections. Virulence. 2015;6(3):196-207.

9. Datta HK, Ng WF, Walker JA, Tuck SP, Varanasi SS. The cell biology of bone metabolism. J Clin Pathol. 2008;61(5):577-87. https://doi.org/10.1136/ jcp.2007.048868.

10. Honda K. Porphyromonas gingivalis sinks teeth into the oral microbiota and periodontal disease. Cell Host Microbe. 2011;10(5):423-5.

11. Hajishengallis G, Lamont RJ. Beyond the red complex and into more complexity: the polymicrobial synergy and dysbiosis (PSD) model of periodontal disease etiology. Mol Oral Microbiol. 2012;27(6):409-19.

12. Hajishengallis $G$, Liang S, Payne MA, et al. Low-abundance biofilm species orchestrates inflammatory periodontal disease through the commensal microbiota and complement. Cell Host Microbe. 2011;10(5):497-506.

13. Lamont RJ, El-Sabaeny A, Park Y, Cook GS, Costerton JW, Demuth DR. Role of the Streptococcus gordonii SspB protein in the development of Porphyromonas gingivalis biofilms on streptococcal substrates. Microbiology. 2002;148(Pt 6):1627-36.

14. Park Y, Simionato MR, Sekiya K, et al. Short fimbriae of Porphyromonas gingivalis and their role in coadhesion with Streptococcus gordonii. Infect Immun. 2005;73(7):3983-9.

15. Daep CA, James DM, Lamont RJ, Demuth DR. Structural characterization of peptide-mediated inhibition of Porphyromonas gingivalis biofilm formation. Infect Immun. 2006;74(10):5756-62.

16. Daep CA, Lamont RJ, Demuth DR. Interaction of Porphyromonas gingivalis with oral streptococci requires a motif that resembles the eukaryotic 
nuclear receptor box protein-protein interaction domain. Infect Immun. 2008;76(7):3273-80.

17. Daep CA, Novak EA, Lamont RJ, Demuth DR. Structural dissection and in vivo effectiveness of a peptide inhibitor of Porphyromonas gingivalis adherence to Streptococcus gordonii. Infect Immun. 2011;79(1):67-74.

18. de Freitas LM, Calixto GM, Chorilli M, et al. Polymeric nanoparticle-based photodynamic therapy for chronic periodontitis in vivo. Int J Mol Sci. 2016;17(5):769.

19. Makadia HK, Siegel SJ. Poly lactic-co-glycolic acid (PLGA) as biodegradable controlled drug delivery carrier. Polymers. 2011;3(3):1377-97.

20. Patel A, Patel M, Yang X, Mitra AK. Recent advances in protein and peptide drug delivery: a special emphasis on polymeric nanoparticles. Protein Pept Lett. 2014;21(11):1102-20.

21. Singh R, Lillard JW Jr. Nanoparticle-based targeted drug delivery. Exp Mol Pathol. 2009;86(3):215-23.

22. de Sousa FF, Ferraz C, Rodrigues LK, Nojosa Jde S, Yamauti M. Nanotechnology in dentistry: drug delivery systems for the control of biofilmdependent oral diseases. Curr Drug Deliv. 2014;11(6):719-28.

23. Elsabahy M, Wooley KL. Design of polymeric nanoparticles for biomedical delivery applications. Chem Soc Rev. 2012:41(7):2545-61.

24. Kalia P, Jain A, Radha Krishnan R, Demuth DR, Steinbach-Rankins JM. Peptide-modified nanoparticles inhibit formation of Porphyromonas gingivalis biofilms with Streptococcus gordonii. Int J Nanomed. 2017;12:4553-62.

25. Steinbach JM. Protein and oligonucleotide delivery systems for vaginal microbicides against viral STIs. Cell Mol Life Sci CMLS. 2015;72(3):469-503.

26. Steinbach JM, Weller CE, Booth CJ, Saltzman WM. Polymer nanoparticles encapsulating siRNA for treatment of HSV-2 genital infection. J Control Release. 2012;162(1):102-10.

27. Martin DT, Steinbach JM, Liu J, et al. Surface-modified nanoparticles enhance transurothelial penetration and delivery of survivin siRNA in treating bladder cancer. Mol Cancer Ther. 2014;13(1):71-81.

28. Sims $L B$, Curtis $L T$, Frieboes HB, Steinbach-Rankins JM. Enhanced uptake and transport of PLGA-modified nanoparticles in cervical cancer. J Nanobiotechnol. 2016;14:33.

29. Steinbach JM, Seo YE, Saltzman WM. Cell penetrating peptide-modified poly(lactic-co-glycolic acid) nanoparticles with enhanced cell internalization. Acta Biomater. 2016:30:49-61

30. Cai X, Han B, Liu Y, Tian F, Liang F, Wang X. Chlorhexidine-loaded amorphous calcium phosphate nanoparticles for inhibiting degradation and inducing mineralization of type I collagen. ACS Appl Mater Interfaces. 2017:9(15):12949-58.

31. Chronopoulou L, Nocca G, Castagnola M, et al. Chitosan based nanoparticles functionalized with peptidomimetic derivatives for oral drug delivery. New Biotechnol. 2016;33(1):23-31.

32. Yao W, Xu P, Pang Z, et al. Local delivery of minocycline-loaded PEG-PLA nanoparticles for the enhanced treatment of periodontitis in dogs. Int J Nanomed. 2014;9:3963-70.
33. Yao W, Xu P, Zhao J, et al. RGD functionalized polymeric nanoparticles targeting periodontitis epithelial cells for the enhanced treatment of periodontitis in dogs. J Colloid Interface Sci. 2015;458:14-21.

34. Benoit DS, Koo H. Targeted, triggered drug delivery to tumor and biofilm microenvironments. Nanomedicine. 2016;11(8):873-9.

35. Lee BS, Lee CC, Wang YP, et al. Controlled-release of tetracycline and lovastatin by poly(D, L-lactide-co-glycolide acid)-chitosan nanoparticles enhances periodontal regeneration in dogs. Int J Nanomed. 2016:11:285-97.

36. Takahashi C, Akachi Y, Ogawa N, et al. Morphological study of efficacy of clarithromycin-loaded nanocarriers for treatment of biofilm infection disease. Med Mol Morphol. 2017;50(1):9-16.

37. Wayakanon $\mathrm{K}$, Thornhill MH, Douglas CW, et al. Polymersome-mediated intracellular delivery of antibiotics to treat Porphyromonas gingivalisinfected oral epithelial cells. FASEB J. 2013;27(11):4455-65.

38. Madhumathi K, Sampath Kumar TS. Regenerative potential and antibacterial activity of tetracycline loaded apatitic nanocarriers for the treatment of periodontitis. Biomed Mater. 2014;9(3):035002.

39. Sindhura Reddy N, Sowmya S, Bumgardner JD, Chennazhi KP, Biswas R, Jayakumar R. Tetracycline nanoparticles loaded calcium sulfate composite beads for periodontal management. Biochem Biophys Acta. 2014;1840(6):2080-90.

40. Lee SF, Hulbah M, Halperin SA. Development of a gene delivery system in Streptococcus gordonii using thymidylate synthase as a selection marker. $J$ Microbiol Methods. 2016;125:43-8.

41. Tiyaboonchai W, Rodleang I, Ounaroon A. Mucoadhesive polyethylenimine-dextran sulfate nanoparticles containing Punica granatum peel extract as a novel sustained-release antimicrobial. Pharm Dev Technol. 2015;20(4):426-32.

42. Moulari B, Lboutounne H, Chaumont JP, Guillaume Y, Millet J, Pellequer Y. Potentiation of the bactericidal activity of Harungana madagascariensis Lam. ex Poir. (Hypericaceae) leaf extract against oral bacteria using poly (D, L-lactide-co-glycolide) nanoparticles: in vitro study. Acta Odontol Scand. 2006;64(3):153-8.

43. Liu L, Shu S, Cheung GS, Wei X. Effect of miR-146a/bFGF/PEG-PEl nanoparticles on inflammation response and tissue regeneration of human dental pulp cells. Biomed Res Int. 2016;2016:3892685.

44. Napimoga MH, da Silva CA, Carregaro V, et al. Exogenous administration of 15d-PGJ2-loaded nanocapsules inhibits bone resorption in a mouse periodontitis model. J Immunol. 2012;189(2):1043-52.

45. Zaltsman N, Ionescu AC, Weiss El, Brambilla E, Beyth S, Beyth N. Surfacemodified nanoparticles as anti-biofilm filler for dental polymers. PLoS ONE. 2017;12(12):e0189397.

\footnotetext{
Ready to submit your research? Choose BMC and benefit from:

- fast, convenient online submission

- thorough peer review by experienced researchers in your field

- rapid publication on acceptance

- support for research data, including large and complex data types

- gold Open Access which fosters wider collaboration and increased citations

- maximum visibility for your research: over 100M website views per year
}

At BMC, research is always in progress.

Learn more biomedcentral.com/submissions 